\title{
ELECTROCATALYTIC ACTIVITY FOR O 2 REDUCTION OF UNSUBSTITUTED AND PERCHLORINATED IRON PHTHALOCYANINES ADSORBED ON AMINO-TERMINATED MULTIWALLED CARBON NANOTUBES DEPOSITED ON GLASSY CARBON ELECTRODES
}

\author{
PAULINA CANEETEI , J. FRANCISCO SILVA, JOSÉ H. ZAGAL* \\ Departamento de Química de los Materiales, Facultad de Química y Biología, Universidad de Santiago de Chile, Casilla 40, correo 33, Santiago, Chile.
}

\begin{abstract}
Amino-functionalized multiwalled carbon nanotubes (MWCNT- $\mathrm{NH}_{2}$ ) were modified with Fe phthalocyanine (FePc) and perchlorinated Fe phthalocyanine $(16(\mathrm{Cl}) \mathrm{FePc})$ and deposited on glassy carbon electrodes (GCE). The electrocatalytic activity of these hybrid electrodes was examined for the reduction of molecular oxygen in alkaline media $(0.2 \mathrm{M} \mathrm{NaOH})$ using stationary and rotating disk electrodes. Electrodes containing $16(\mathrm{Cl}) \mathrm{FePc}$ are more active than those containing FePc. Electrodes containing CNTs are more active than electrodes without CNTs but the higher activity can be attributed to a greater real surface area, compared CGEs and not necessarily to a catalytic effect.
\end{abstract}

Keywords: Amino-terminated carbon nanotubes, Fe phthalocyanines, $\mathrm{O} 2$ reduction, catalytic activity.

\section{INTRODUCTION}

MN4 macrocyclic metal complexes are well known as catalyst for many electrochemical reactions, specially the reduction of $\mathrm{O}_{2}$ and their activity is directly related to their redox properties ${ }^{6,7}$. On the other hand, carbon nanotubes (CNTs) are interesting support materials for the modification of substrates ${ }^{8}$. CNTs increase the area of the electrodes and when this is combined with MN4 macrocyclic complexes substantial improvements in the reactivity $7,9-13$ are observed. The specific chemistry of the surface of CNTs can be tailored by changing the nature of surface groups that can be created on their surface by appropriate chemical treatment, adapting them for a desired application ${ }^{8-13}$. A non-chemical way of modification of CNTs is by simple adsorption of macrocyclic complexes on the external walls of the CNTs where the molecules stick to them by $\pi-\pi$ interactions. If carbon nanotubes are modified with amino groups $-\mathrm{NH}_{2}$, they can coordinate to the axial position of the $\mathrm{MN}_{4}$ complex ${ }^{13}$. The reduction of $\mathrm{O}_{2}(\mathrm{ORR})$ is the most studied reaction using MN4 complexes as catalysts, with the aim of replacing expensive Pt containing materials in the cathode of fuel cells ${ }^{14}$. In aqueous media ORR can proceed via two main pathways: via the transfer of $2-\mathrm{e}^{-}$to give peroxide and via $4 \mathrm{e}^{-}$to give water. The 4-e- reduction of $\mathrm{O}_{2}$ involves the breaking of the $\mathrm{O}-\mathrm{O}$ bond and delivers more energy than the two-e- reduction ${ }^{15}$. In alkaline media, Fe phthalocyanines promote the $4 \mathrm{e}^{\mathrm{e}}$ reduction of $\mathrm{O}_{2}$ almost entirely to water ${ }^{11}$. In this manuscript we have examined the ORR reaction in alkaline media $(0.2 \mathrm{M} \mathrm{NaOH})$ using as catalysts $\mathrm{Fe}$ phthalocyanine $(\mathrm{FePc})$ and perchlorinated $\mathrm{Fe}$ phthalocyanine $(16(\mathrm{Cl}) \mathrm{FePc})$ and incorporated on amino-terminated multiwalled carbon nanotubes.

\section{EXPERIMENTAL}

Iron phthalocyanine $(\mathrm{FePc})$ and iron hexadecachlorophthalocyanine $16(\mathrm{Cl}) \mathrm{FePc}$ were obtained from Aldrich. Amino-functionalized multiwalled carbon nanotubes $(\mathrm{amCNT})$ were purchased from DropSens. N,N-dimethyl formamide (DMF) was obtained from Merck. High purity $\mathrm{N}_{2}$ and $\mathrm{O}_{2}$ gases were obtained from Indura. All other reagents were of analytical grade and were used as received.

Prior to electrode modification different amCNT/FePcs dispersions were prepared. The amCNT was dispersed in $1 \mathrm{mgmL}^{-1}$ of the complex with the aid of ultrasonic stirring for $30 \mathrm{~min}$. The dispersion was then left at room temperature for $24 \mathrm{~h}$. The amCNT/FePcs dispersions were filtered and thoroughly washed with DMF and ethanol. The solid was dried at $40{ }^{\circ} \mathrm{C}$ for $24 \mathrm{~h}$. The working electrode was a glassy carbon electrode disk (GCE, Pine instruments) of 0.196 $\mathrm{cm}^{2}$. The GCE was polished with 2400 and 4000 grit emery paper followed by ultrasonic treatment in purified water for $1 \mathrm{~min}$. In order to modify the GCE $1 \mathrm{mg}$ of the dry amCNT/FePcs was dispersed in $1 \mathrm{~mL}$ DMF and sonicated for 15 min. $5 \mu \mathrm{L}$ of the amCNT/FePcs dispersion was cast on the GCE surface and allowed to dry at $60{ }^{\circ} \mathrm{C}$ for $15 \mathrm{~min}$. Electrochemical measurements were conducted on an Epsilon Voltammetric Analyzer. An ASR Pine rotator unit was used for the rotating disk electrode experiments., The counter electrode was a platinum spiral wire $(99.99 \%$, Aldrich), and a $\mathrm{Ag} / \mathrm{AgCl}$ electrode saturated with $\mathrm{KCl}$ was the reference electrode. Measurements were conducted at $25{ }^{\circ} \mathrm{C}$. wit $0.2 \mathrm{M} \mathrm{NaOH}$ was prepared using deionized, bidistilled water. CV curves were obtained in $\mathrm{N}_{2}$ saturated solutions and polarization curves with $\mathrm{O}_{2}$ saturated solutions.

\section{RESULTS AND DISCUSSION}

The structure of phthalocyanine $(\mathrm{FePc})$ and hexadecachloro $\mathrm{Fe}$ phthalocyanine $16(\mathrm{Cl}) \mathrm{FePc}$. Figure 2A compares two cyclic voltammograms obtained in $0.2 \mathrm{M} \mathrm{NaOH}$, one, illustrating the response of the bare glassy carbon electrode and then, after the incorporation of the CNT nanotubes (GCE/ amCNT). There are no faradaic currents in the potential region examined. The only effect of the addition of the carbon nanotubes is the increase in the capacitive currents by a factor or almost two, which reflects the increase in the real surface area of the electrode interface by the presence of the nanotubes. The dashed lines in Figure 2A illustrate the cyclic voltammograms obtained after modifying the amCNTs with $\mathrm{FePc}$ (GCE/amCNT/FePc). There is an increase in the capacitive currents by the presence of FePc and three redox peak appear $-0.68-0.49$ and $-0.22 \mathrm{~V}$ respectively. These peaks are more clearly visible in Figure $\mathrm{AB}$ that compares the square wave voltammograms of the same electrodes, i.e. the GCE/amCNT/FePc and GCE/amCNT. It is clear in Figure $3 \mathrm{~B}$ that the electrode having unmodified carbon nanotubes does not show any faradaic currents in the potential region examined. The redox peaks at $-0.49 \mathrm{~V}$ and $-0.22 \mathrm{~V}$ have been assigned to the $\mathrm{Fe}(\mathrm{II}) /(\mathrm{I})$ and $\mathrm{Fe}(\mathrm{III}) /(\mathrm{II})$ reversible processes respectively. The electrical charge under each peak is ca. $5.8 \times 10^{-6} \mathrm{C}$. One can estimate the amount of molecules by dividing the charge by the Faraday constant that is $96,485 . \mathrm{C} \mathrm{mol}^{-1}$ and this gives a value of $0.6 \mathrm{x}$ $10^{-10} \mathrm{~mol}$ or $3.06 \times 10^{-10} \mathrm{~mol} \mathrm{~cm}^{-2}$ using the geometric area of the electrode. It is important to point out that the electrode is not smooth so this gives a rough idea of the amount of molecules present on the carbon nanotubes. However, Figure 2A shows that the capacitive currents under the presence of the carbon nanotubes increase by a factor of two so, the real surface area of the electrode is at least twice the geometric area of $0.196 \mathrm{~cm}^{2}$.

Figure 3 shows similar results, but obtained with $16(\mathrm{Cl}) \mathrm{FePc}$. The redox potentials for both $\mathrm{Fe}(\mathrm{II}) /(\mathrm{I})$ and $\mathrm{Fe}(\mathrm{III}) /(\mathrm{II})$ reversible processes are shifted to more positive potentials for $16(\mathrm{Cl}) \mathrm{FePc}$ compared to FePc. For example the $\mathrm{Fe}(\mathrm{II}) /(\mathrm{I})$ moves $0.21 \mathrm{~V}$ in the positive direction by the effect of the $-\mathrm{Cl}$ groups whereas the $\mathrm{Fe}(\mathrm{III}) /(\mathrm{II})$ redox pair moves $0.13 \mathrm{~V}$, so the $\mathrm{Fe}(\mathrm{II}) /(\mathrm{I})$ redox process is more sensitive to the effect of the $-\mathrm{Cl}$ groups than the $\mathrm{Fe}(\mathrm{III}) /(\mathrm{II})$ redox process. The intensity of the peaks in Figure $3 \mathrm{~B}$ attributed to the presence of $16(\mathrm{Cl}) \mathrm{FePc}$ are more intense compared to those observed with unsubstituted $\mathrm{FePc}$. It is possible that the more electron deficient $\mathrm{Fe}$ center in $16(\mathrm{Cl}) \mathrm{FePc}$ coordinates better to the amino groups present on the CNTs than FePc, so this could explain the higher coverages observed with $16(\mathrm{Cl}) \mathrm{FePc}$ compared to FePc. The electrical charge under each peak is ca. $20.0 \times 10^{-6} \mathrm{C}$ and this gives 
a value of $2.07 \times 10^{-10} \mathrm{~mol}$ or $10.56 \times 10^{-10} \mathrm{~mol} \mathrm{~cm}^{-2}$, again using the geometric area of the electrode. Compared to FePc, the surface coverage of $16(\mathrm{Cl}) \mathrm{FePc}$ is about 3.44 times higher.
A



B

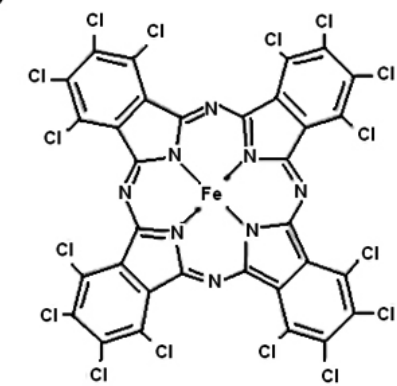

Figure 1. Structures of $\mathrm{Fe}$ phthalocyanines used in this study: (A) Unsubstituted Fe phthalocyanine (FePc) (B) Fe hexadecachloro phthalocyanine $\left(\mathrm{FeCl}_{16} \mathrm{Pc}\right)$.
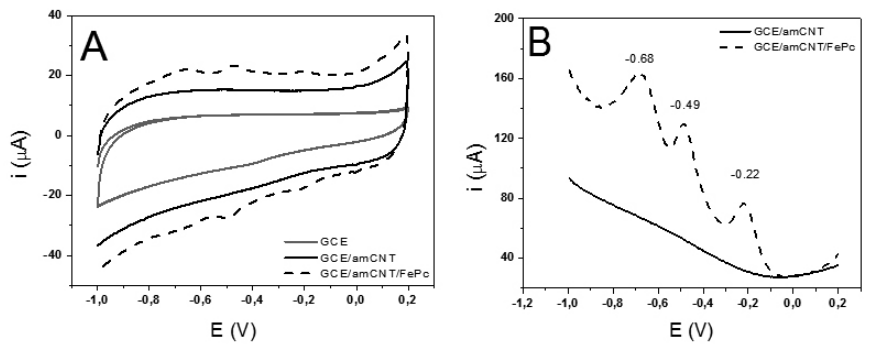

Figure 2. (A) Cyclic voltammograms of bare and modifiedGCE with amCNT and amCNT/FePc, in NaOH $0.2 \mathrm{M}(v=100 \mathrm{mV} / \mathrm{s})$. (B) Square wave voltammetry of GCE/amCNT and GCE/amCNT/FePc. Parameters: amplitude $25 \mathrm{mV}$, frequency $15 \mathrm{~Hz}$.
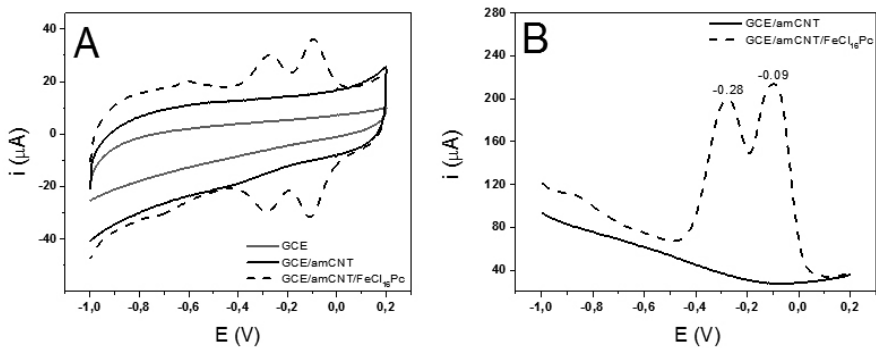

Figure 3. (A) Cyclic voltammograms of bare (grey) and modifiedGCE with amCNT and amCNT/ $\mathrm{FeCl}_{16} \mathrm{Pc}$, in $\mathrm{NaOH} 0.2 \mathrm{M}(v=100 \mathrm{mV} / \mathrm{s})$. (B) Square wave voltammetry of $\mathrm{GCE} / \mathrm{amCNT}$ and $\mathrm{GCE} / \mathrm{amCNT} / \mathrm{FeCl}{ }_{16} \mathrm{Pc}$. Parameters: amplitude $25 \mathrm{mV}$, frequency $15 \mathrm{~Hz}$.

Figure 4 compares ORR rotating-disk polarization curves for 4 different types of electrodes namely:GCE, GCE/amCNT, CGE/amCNTFePc and CGE/ amCNT/16(Cl)FePc. The response of the bare GCE is known and essentially corresponds to the reduction of $\mathrm{O}_{2}$ via $2 \mathrm{e}^{-}$to give $\mathrm{HO}_{2}^{-}$. The larger currents obtained with the GCE/amCNT electrode can be attributed almost entirely to an area effect, even though some catalytic effect of CNTs could be expected as the foot of the reduction wave is shifted by $0.1 \mathrm{~V}$ in the positive direction. After adding FePc to the CNTs the catalytic effect is evident since the currents increase by a factor of two or more and the foot of the wave is shifted in the positive direction by $0.3 \mathrm{~V}$ compared to bare GCE and by ca. $0.2 \mathrm{~V}$ compared to the GCE/amCNT electrode. The duplications of the diffusion limiting currents are attributed to a change in mechanism from a transfer of two electrons to a transfer of 4-electrons. It is know that $\mathrm{FePc}$ promotes the 4-electron reduction of $\mathrm{O}_{2}$ in alkaline media ${ }^{1}$. The presence of $16(\mathrm{Cl}) \mathrm{FePc}$ produces even larger currents and a shift in the foot of the wave in the positive direction. It is well established form previous work that electron-withdrawing substituents on the phthalocyanine ligand favor the $\mathrm{O}_{2}$ reduction process so this is not surprising. In fact the more positive the $\mathrm{Fe}(\mathrm{III}) /(\mathrm{II})$ redox potential, the better for the catalytic activities of phthalocyanines ${ }^{1,7}$. The large currents of the GCE/amCNT/16(Cl) $\mathrm{FePc}$ electrode compared to $\mathrm{GCE} / \mathrm{amCNT} / \mathrm{FePc}$ can then be attributed to two factors namely, the more positive $\mathrm{Fe}(\mathrm{III} /(\mathrm{II})$ redox potential of $16(\mathrm{Cl}) \mathrm{FePc}$ compared to $\mathrm{FePc}$ and the higher surface coverage of $16(\mathrm{Cl}) \mathrm{FePc}$ on the CNTs compared to FePc. It is difficult to separate these two effects since it is possible that the $16(\mathrm{Cl}) \mathrm{FePc}$ molecules form multilayers on the CNTs and it is expected that only the outermost molecules will be accessible to $\mathrm{O}_{2}$. So the larger surface concentration of $16(\mathrm{Cl}) \mathrm{FePc}$ may not necessarily be reflected in a higher catalytic activity. We are currently investigating the possibility of preparing amCNTs with different surface concentrations of $16(\mathrm{Cl}) \mathrm{FePc}$ and see if this affects the catalytic currents for ORR.

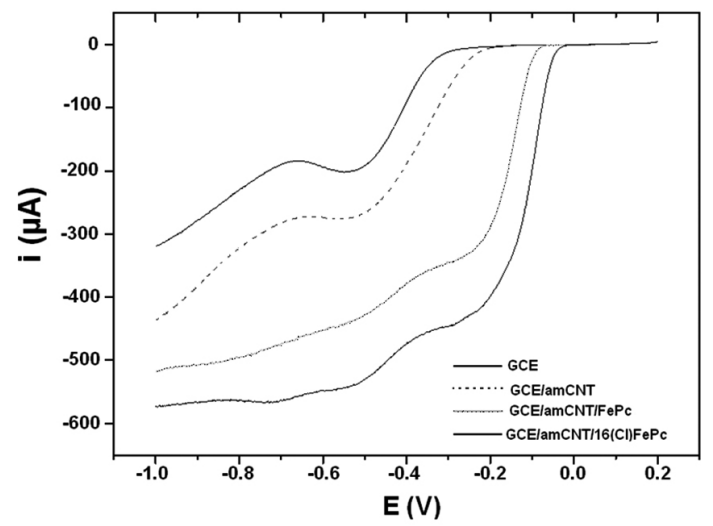

Figure 4. Polarization curves for ORR for a GCE (solid line) GCE/ amCNT (dashed line), GCE/amCNT/FePc (dotted line) GCE/amCNT/16(Cl) FePc with a rotating disk electrode in $\mathrm{NaOH} 0.2 \mathrm{M} \mathrm{O}_{2}$ saturated $\left(v=5 \mathrm{mVs}^{-1}\right.$, $\omega=1000 \mathrm{rpm})$.

\section{ACKNOWLEDGEMENTS}

Work supported by Fondecyt Projects 1140199, Millennium Nucleus of Molecular Engineering for Catalysis and Biosensors RC120001 and Dicyt USACH P.C. thanks a postdoctoral position of the Millennium Nucleus.

\section{REFERENCES}

1. J. H. Zagal, S. Griveau, J.F. Silva, T. Nyokong, F. Bedioui, Coord. Chem. Revs. 254, 2755, (2010)

2. J. Masa, W. Schuhmann, Chem. Eur. J. 19, 9644, (2013)

3. J.H. Zagal, P. Cañete, J. Recio, F. Tasca, C. Linares-Flores, Electrochem. Commun. 30, 34, (2013)

4. F. Bedioui, S. Griveau, T. Nyokong, A.J. Appleby, C.A. Caro, M. Gulppi, G.Ochoa, J.H. Zagal, Phys. Chem. Chem. Phys. 9, 3383, (2007)

5. S. Maree, T. Nyokong, J. Electroanal. Chem. 492, 120, (2000).

6. J.H.Zagal, F. J. Recio, C. A. Gutiérrez, C.Zúñiga, M. A. Páez. Electrochem. Comm. 41, 24 (2014)

7. C. Gutierrez, J. F. Silva, F. J. Recio, S. Griveau, F. Bedioui, C. A. Caro, J. H. Zagal, Electrocatalysis (2014), doi.org/10.1007/s12678-014-0209-y

8. A. Dumitru, M. Mamlouk, K. Scott, Electrochim Acta. 134, 428, (2014)

9. A. Okunola, B. Kowalewska, M. Bron, P. J. Kulesza, W. Schuhmann, Electrochim. Acta. 54, 1954, (2009)

10. F. Moraes, M. F. Cabral, S. Machado, L. Mascaro, Electroanalysis. 20, $851,(2008)$

11. F. Moraes, L. Mascaro, S. Machado, C. Brett, Talanta, 79, 1406, (2009)

12. T. Schilling, A. Okunola, J. Masa, W. Schuhmann, M. Bron, Electrochim. Acta. 55, 7597, (2010)

13. M. Sancy, J. Francisco Silva, J. Pavez, J. H. Zagal, J. Chil. Chem. Soc. 58, $1842,(2013)$

14. J.K. Nørskov, J. Rossmeisl, A. Logadottir, L. Lindqvist, J.R. Kitchin, T. Bligaard, H. Jonsson, Phys. Chem. B. 108, 17886, (2004)

15. J.H. Zagal, M. Paez and J F.Silva in N4-Macrocyclic Metal Complexes, eds. J. H. Zagal, F. Bedioui and J. P. Dodelet, Springer, New York, 2006, pp. 41 